International Journal of Forensic Science \& Pathology (IJFP)

ISSN 2332-287X

\title{
Medicolegal Consequences of Death due to Stevens Johnson Syndrome: A Case Report
}

\author{
Laxman $G^{1 *}$, S.K.Meshram² ${ }^{2}$ S.G,Dhawane ${ }^{2}$, V.N.Ambade ${ }^{2}$, P.G.Dixit ${ }^{3}$
}

\author{
Case Report
}

${ }^{1}$ Resident Doctor, Department of Forensic medicine, Govt. Medical College and Hospital, Nagpur, India.
${ }^{2}$ Associate Professor, Department of Forensic medicine, Govt. Medical College and Hospital, Nagpur, India.
${ }^{3}$ Professor and Head, Department of Forensic medicine, Govt. Medical College and Hospital, Nagpur, India.

\begin{abstract}
Conditions like Stevens Johnson syndrome are very rare and mortality arising out of it is still a rarest entity. It is immune complex mediated hypersensitivity reaction; most common factors to trigger the immunity are drugs, followed by infection with various organisms, which starts the chain of events in SJS. It sometimes associated with HLA Antigen and 25-50\% cases are idiopathic.
\end{abstract}

In present case an emphasis is given on progression of this life threatening condition, mode of death, responsibilities of the physician and legal as well as criminal consequences arise out of it while treating such cases.

Keywords: Stevens Johnson Syndrome; Hypersensitivity Reaction; Erythema Multiforme.

\section{*Corresponding Author:}

Laxman GangadharPhad,

Resident Doctor, Department of Forensic medicine, Govt. Medical College and Hospital, Room no15, New PG Hostel, Nagpur - 440003, India. Tel: 9421381771/8805523842

E-mail: laxmanphad87@gmail.com

Received: March 14, 2015

Accepted: April 30, 2015

Published: May 12, 2015

Citation: Laxman G, S.K. Meshram, S.G. Dhawane, V.N. Ambade, P.G. Dixit (2015) Medicolegal Consequences of Death due to Stevens Johnson Syndrome: A Case Report. Int J Forensic Sci Pathol. 3(5), 123-126. doi: http://dx.doi.org/10.19070/2332-287X-1500030

Copyright: Laxman $\mathbf{G}^{\circ}$ 2015. This is an open-access article distributed under the terms of the Creative Commons Attribution License, which permits unrestricted use, distribution and reproduction in any medium, provided the original author and source are credited.

\section{Introduction}

Stevens Johnson syndrome (SJS) has a potential to cause severe morbidity and mortality, having incidence of 1-6 cases per million person years [1]. It was firstly described in 1922 by A.M. Stevens and Frank C. Johnson in pediatric population. It is idiosyncratic immune complex mediated hypersensitivity reaction and fatal form of erythema multiforme. There is symptomatic involvement of skin and mucous membrane of oral, nasal, ocular, vaginal, urethral, gastrointestinal and respiratory tract.

There is no reliable test exists to establish a link between a particular drug and SJS in an individual case [2]. Determining the causative drug is based on the time interval between the use of drug and the beginning of the skin reaction. Typically the symptoms of drug-induced SJS arise within a week of starting the medication
[3]. People with systemic lupus erythematous or HIV infections are more susceptible to drug-induced SJS [3]. The second most common cause of SJS is infection, but the routine use of medicines such as antibiotics, antipyretics and analgesics to manage infections make it difficult to determine, whether the events started by infection or medicines taken [4].

The pathogenesis of SJS is multifactorial and is probably due to a dynamic interplay between acquired and constitutional factors in the presence of threshold amount of the drug or its metabolites. Inability to detoxify the intermediate drug metabolites which may serve as haptens when complexes with host epithelial tissue could initiate an immune reaction. Once initiated various triggers propagate the immune response leading to keratinocyte apoptosis, a wide variety of causative drugs have been identified and these are related to the spectrum of local disease and local prescribing practices [5]. SJS is strongly associated with HLA-B*1502 (HLAB75), an HLA-B serotype of the broader serotype HLA-B15 [6, 7]. A study in Europe suggested the gene marker is only relevant for East Asians [8].

\section{Case Report}

A 16 year old unmarried girl was brought for postmortem examination in forensic medicine dept. GMCH Nagpur. The details of case were collected from police investigation records, relatives and hospital treatment records.

She had complaints of fever and seizures on day 1. Relatives taken her to nearby private hospital; physician started the treatment with paracetamol and phenytoin. Then she starts to develop fever, cough, and rashes from day 2 onwards. But treating physician continued the treatment, he neither taken consultation of other expert (dermatologist) nor discontinued the treatment, but still assured the relatives regarding the cure of patient. By the time, 
Rashes gradually started to progress on face and upper chest, which is followed by blistering of skin, the condition of patient is worsening day by day. The rashes progressed to exfoliation of skin, so the treating physician absconded for two days leaving the patient in such a critical condition without discharging the patient, without informing the relatives and without handover the patient to compatible doctor. So relatives shifted the patient to other hospital by themselves. Where the diagnosis was made as a Stevens Johnson syndrome. But due to lack of facilities, doctor referred the patient to multispecialty hospital on day 17.

On admission pulse-150/min, laryngeal edema present so patient intubated with endotracheal tube and kept on ventilator for five days, then endotracheal tube is removed on day 22 , and continued supportive treatment with skin care and I.V. fluids till day 30. Then she develops difficulty in breathing so tracheostomy done and kept her on ventilator on Day 31. Laboratory Investigation shows Leucopenia, Neutrophilia, serum Na+-156 Mmol/L, serum K+-3.3Mmol/L, Hb-8.7gm\%, SGOT- $331 \mathrm{IU} / \mathrm{L}$, SSPT - 196IU/L, Total protein- 4.6gm/dl. Blood culture \& sensitivity shows infection with Candida and Acinetobacter baumannii, which are resistant to all broad spectrum antibiotics except collistin and tigecyclin. In spite of adequate treatment Patient died on day $34^{\text {th }}$.

Immediately after death, the relatives lodge the complaint of negligence against the first treating physician. The medico-legal autopsy carried out at GMCH, Nagpur.

\section{Postmortem Examination}

\section{External Examination}

Skin shows application of bright red ointment over the skin area.

Exfoliated skin lesions present all over the body except over the scalp, it is completely involving skin of face, neck, trunk, left upper limb up to half arm, and right upper limb up to elbow, up to knees on both side and rest of skin is involved in patchy areas of brownish irregular slightly raised plaques. On dissection, infiltration of blood is seen in soft tissues.

Tracheostomy wound present over anterior aspect of neck at midline, $1 \mathrm{x} 1 \mathrm{~cm}$ oozing mucoid, frothy discharge.

\section{Internal Examination}

Yellowish discoloration present at under scalp region, with thickening and yellowish discoloration of meninges, brain is soft and edematous (weight: $1270 \mathrm{gm}$.).

Lungs (Right: $887 \mathrm{gm}$, Left: $721 \mathrm{gm}$ ) are heavy, edematous and congested. Lower lobes of both lungs shows consolidation with red hepatisation, on cut section frothy fluid oozes out. Buccal mucosa shows patchy ulceration, esophagus, stomach and small intestinal mucosa shows ulceration and erosion at places. Spleen

Figure 1. Showing exfoliated skin lesions and peeling of skin all over the trunk with application of reddish coloured ointment.

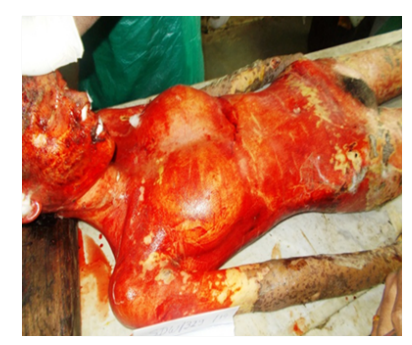

Figure 2. Showing involvement of skin in patchy areas over lower limbs and part of upper limbs.

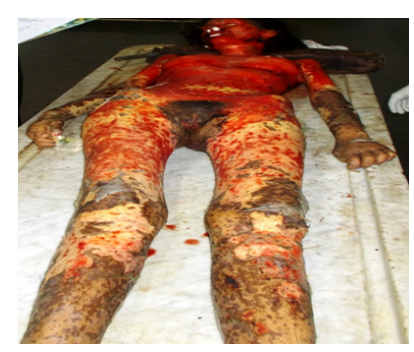

Figure 3. showing patchy involvement of skin with spared areas over legs.

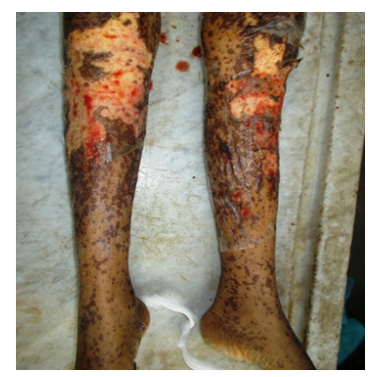


Figure 4. showing splenomegaly with subcapsular pus pockets at places.

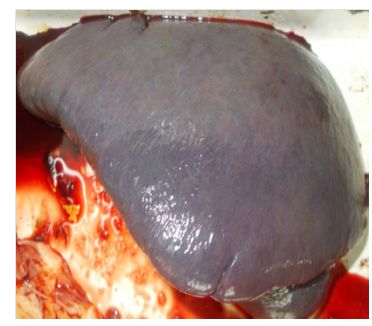

Figure 5. showing pulmonary odema, frothy fluid oozes out on cut section.

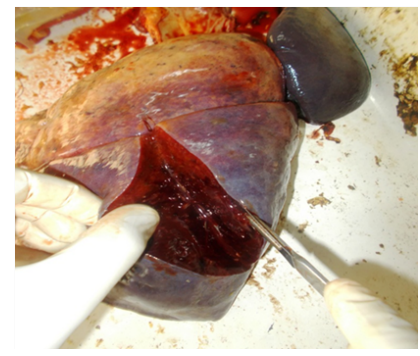

Figure 6. showing yellowish discoloration of liver with hepatomegaly.

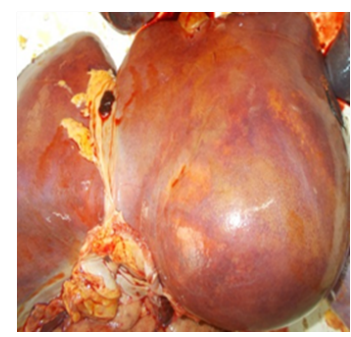

Figure 7. showing histopathological changes in Stevens Johnson syndrome in skin sample.

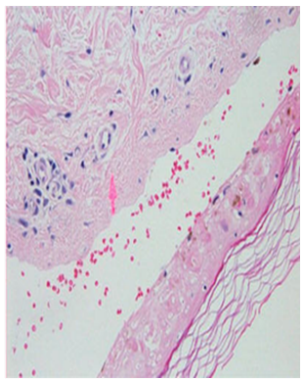

(600gm.) shows Subcapsular pus pockets at places, with splenomegaly. Heart (244gm) congested, Liver (1690 gm.) shows yellowish discoloration, and hepatomegaly, Kidneys (Right: 195gm, Left: $190 \mathrm{gm}$.) are congested.

Histopathological reports of skin sample shows, edema, with lymphocytic infiltration at the dermoepidermal junction, and migration of lymphocyte towards epidermis and necrosis at epidermal site.

Cause of death is given as death Due to "septicemia associated with multiorgan failure in a diagnosed case of SJS."

\section{Discussion}

The SJS is immune complex mediated hypersensitivity reaction involving skin and mucous membrane; various etiological factors trigger the immunity, such as infections with viruses, bacteria, protozoa, and some fungi. Drugs like sulfa preparations, Analgesics, Phenytoin, Antiretroviral, Antitubercular and many others. The disease starts from simple rash, which progressed into papule, papule gets converted into vesicle and bullous lesion, which then ruptured to form denudation of skin which further gets ulcerated and necrosed, which finally ends in slippage of skin due to death of keratinocyte. If the Body surface area (BSA) involvement is $<10 \%$, the mortality rate is up to $1-5 \%$, but it may raise up to $25-50 \%$ if BSA involvement is $>30 \%$. Some famous personalities who are diagnosed and died of SJS are Padma Lakshmi, actress and model, television personality, and cook book writer [10], Tessa Keller of MTV show [9], ManuteBol, former professional basketball player and member of NBA's Washington Bullets [11], Gene Sauers, three-time PGA Tour winner [12], Calvin Lock, from Littleport, Cambridgeshire [9].

In present case deceased had signs of hepatic failure like yellowish discoloration of sclera under scalp region meninges liver and other organs. Signs of pulmonary edema and consolidation are present, lungs are heavy and voluminous, brain is edematous and soft, and mucosa of gastrointestinal tract is ulcerated and eroded. This indicates that the patient has entered into multiorgan failure stage. The histopathological finding of skin shows edema of lesion lymphocytic infiltration at the dermoepidermal junction, later on migration of lymphocyte towards epidermis. This profile exactly matches the Histopathological profile of this patient.Now 
the question arises, which drug has stated the chain of disease? She has received two drugs before starting the rashes i.e. analgesic and phenytoin. She might have taken the analgesics many of the times in her 16 years of life, but she had not developed such a reaction, it was her first time of suffering from convulsions and receiving medicines for that, so most probable drugis phenytoin.

After thorough study of the case papers, it was concluded that first treating physician had started treatment with analgesics and antiepileptic drugs for fever and seizers. These drugs are most important in causing SJS. After the development of rashes and blisters he was continued the same treatment and unable to suspect SJS. Also the treating physician had not taken consultation of dermatologist or other doctor, even after having sufficient time and facility, moreover when the condition of patient is serious; the doctor absconds for two days without prior Intimation to the relatives. Hence in prima facie, there is dereliction of duty and deficiency in services provided by the first treating physician cannot be ruled out. Allegations were made by relatives against the first treating physician regarding the negligence in the treatment. The final opinion will be judged by panel of expert doctors appointed.

\section{Conclusion}

By considering the postmortem findings the presence of septicemia in the deceased is due to loss of skin which is the most important in checking the infection. And the signs of multi organ failure are present like brain, lungs, liver spleen. These two parameters contributed to the death of deceased. So the final cause of death is "septicemia associated with multiorgan failure in a diagnosed case of SJS."

\section{References}

[1]. http://bestpractice.bmj.com/best-practice/welcome.html, Last updated: Aug 06,2014

[2]. Mockenhaupt M (2011) "The current understanding of Stevens-Johnson syndrome and toxic Epidermal necrolysis". Expert Review of Clinical Immunology 7(6): 803-15.

[3]. HeleneTigchelaar, NirupamaKannikeswaran, Deepak M. Kamat (2008) Stevens-Johnson Syndrome Pediatric Skin Diseases, Bacterial Conjunctivitis. http://www.pediatricsconsultantlive.com

[4]. Bentley John, Sie, David (2014) "Stevens-Johnson syndrome and toxic epidermal necrolysis". The Pharmaceutical Journal 293 (7832).

[5]. RannakoeLehloenya (2007) Management of Stevens-Johnson syndrome and toxic epidermal necrolysis, hospital and University of Cape Town, south Africa. Current Allergy \& Clinical Immunology 20(3): 124-128.

[6]. Chung WH, Hung SI, Hong HS, Hsih MS, Yang LC, et al. (2004) "Medical genetics: A marker for Stevens-Johnson syndrome". Brief Communications Nature 428(6982): 486.

[7]. Locharernkul C, Loplumlert J, Limotai C, Korkij W, Desudchit T, et al.(2008) "Carbamazepine and phenytoin induced Stevens-Johnson syndrome is associated with HLA-B*1502 allele in Thaipopulation". Epilepsia 49(12): 2087-91.

[8]. Alfirevic A, Jorgensen AL, Williamson PR, Chadwick DW, Park BK, et al. (2006) "HLA-B locus in Caucasian patients with carbamazepine hypersensitivity". Pharmacogenomics 7(6): 813-8

[9]. http://en.wikipedia.org/wiki/Main_Page.

[10]. Cartner-Morley, Jess (2006) "Beautiful and Damned". The Guardian.

[11]. ManuteBol dies at age 47". FanHouse. AOL. 19 June 2010. Archived from the original on 2010-06-21.

[12]. Graff, Chad (2013) "3M golf: Gene Sauers thriving after torturous battle with skin disease". St. Paul Pioneer Press. 